\title{
EMBEDDING TOPOLOGICAL SEMIGROUPS IN TOPOLOGICAL GROUPS
}

\author{
by SHEILA A. MCKILLIGAN \\ (Received 12th September 1969)
}

\section{Introduction}

If we consider a semigroup, its algebraic structure may be such that it is isomorphic to a subsemigroup of a group, or is algebraically embeddable in a group. This problem was investigated in 1931 by Ore who obtained in (4) a set of necessary conditions for this embedding. A necessary condition is that the semigroup should be cancellative: for any $a, x, y$ in the semigroup either $x a=y a$ or $a x=a y$ implies that $x=y$. Malcev in (3) showed that this was not sufficient. It is enough to note that his example was a non-commutative semigroup: a commutative cancellative semigroup is embeddable algebraically in a group.

If, however, we give a semigroup $S$ a Hausdorff topology and require the multiplication to be a continuous map from $S \times S$ to $S$, so that $S$ is a topological semigroup, then an obvious question is whether or not $S$ can be embedded topologically in a topological group. In other words, is $S$ topologically isomorphic to a subsemigroup of a topological group? This question has been investigated in (2), (6), (8), (9) and (10).

It is the purpose of this paper to answer a slightly narrower question, namely, when is a topological semigroup embeddable in a group as a subsemigroup with non-empty interior? This problem has been considered in (7) but the solution given there seems too complicated to be useful. A much simpler solution has been given in (5) (Theorem 3.2.14) but there is a gap in the proof and the result is false as stated (see Example 5.3 below). In this paper we effectively complete the result of (5) and consider some related questions.

I should like here to express my gratitude to my research supervisor, $\mathrm{Dr} \mathrm{A}$. J. White, to whom I am indebted for encouragement and invaluable advice. Acknowledgement is also due to the Carnegie Trust for their generous financial support while this work was being done.

\section{Embedding in the group of quotients}

Let $S$ be a cancellative semigroup such that $a S \cap b S \neq \varnothing$ for $a, b \in S$. It was shown by Ore in (4) that $S$ may be embedded algebraically in a group as follows. An equivalence relation $R$ is defined on $S \times S$ by $(a, b) R(c, d)$ if 
$b x=d y$ for some $x, y \in S$ implies that $a x=c y$. It is clear that $R$ is reflexive and symmetric and, since $a S \cap b S \neq \varnothing$ for $a, b \in S, R$ is also transitive. The set of equivalence classes is denoted by $Q(S)$ and multiplication is defined on $Q(S)$ by $[a, b][c, d]=[a s, d t]$ where $s, t \in S$ are such that $b s=c t$. The group $Q(S)$ with this operation is called the group of quotients of $S$. For any $p \in S$,

$$
[x q, q]=[x p, p] \quad(q \in S, x \in S) .
$$

Consequently, if $p \in S$, the equation

$$
P(x)=[x p, p] \quad(x \in S)
$$

defines a homomorphism $P$, of $S$ into $Q(S)$, which is independent of the choice of $p$. Since $P$ is one-one, $P$ embeds $S$ in $Q(S)$. If $S$ is a topological semigroup, $Q(S)$ has a natural topology: that obtained by endowing $S \times S$ with the product topology and $Q(S)$ with the quotient topology from the relation $R$. This will be referred to as the quotient topology of $Q(S)$.

It will often be convenient to identify the image of $S$ in $Q(S)$ with $S$ itself. A point $s \in S$ then appears as $s$ in $Q(S)$, its inverse as $s^{-1}$, and every element of $Q(S)$ may be written in the form $s t^{-1}$ where $s, t \in S$. A set $A \subset Q(S)$ is open in the quotient topology if $A^{*}=\left\{(x, y): x, y \in S\right.$ and $\left.x y^{-1} \in A\right\}$ is open in $S \times S$.

Our first theorem shows that the quotient topology is the strongest topology which we can assign to $Q(S)$ and for which embeddability is possible.

Theorem 2.1. Let $S$ be a cancellative topological semigroup such that $a S \cap b S \neq \varnothing$ for $a, b \in S$. If $S$ is embeddable in a topological group $G$, then the topology which $Q(S)$ inherits as a subspace of $G$ is weaker than the quotient topology of $Q(S)$.

Proof. Since $Q(S)$ is a topological group with the topology which it inherits from $G$, we treat the case $G=Q(S)$. Let $Q(S)$ have the uniformity with basis $\mathscr{B}$ consisting of all sets $B_{U}=\left\{(x, y): x, y \in Q(S)\right.$ and $\left.x y^{-1} \in U\right\}$ for $U$ a symmetric neighbourhood of the identity of $Q(S)$. Then a uniformity which generates the topology of $S$ has basis $\mathscr{B}_{S}=\left\{B_{U} \cap(S \times S): B_{U} \in \mathscr{B}\right\}$.

Suppose $A \subset Q(S)$ is open in the uniform topology. We now show that $A^{*}=\left\{(s, t): s, t \in S\right.$ and $\left.s t^{-1} \in A\right\}$ is open in $S \times S$. Choose $(s, t) \in A^{*}$. Then $s t^{-1} \in A$ and there exists $B_{U} \in \mathscr{B}$ such that $s t^{-1} \in s t^{-1} B_{U}[e] \subset A$, where $e$ is the identity of $Q(S)$. Choose $B_{V} \in \mathscr{B}$ such that $B_{V} \circ B_{V} \subset B_{U}$. If $W=s^{-1} V s$ and $(p, q) \in\left[B_{V}[s] \times\left(B_{W}\left[t^{-1}\right]\right)^{-1}\right] \cap(S \times S)$, then $(p, s) \in B_{V}$ and $\left(q^{-1}, t^{-1}\right) \in B_{W}$. By the construction of $W,\left(s q^{-1}, s t^{-1}\right) \in B_{V}$ and, since $\left(p q^{-1}, s q^{-1}\right) \in B_{V}$, $\left(p q^{-1}, s t^{-1}\right) \in B_{U}$ from which it follows that $p q^{-1} \in s t^{-1} B_{U}[e] \subset A$. Hence $(p, q) \in A^{*}$, i.e. $A^{*}$ is open in $S \times S$.

The principal result of this section shows that the problem of embedding a topological semigroup $S$ as an open subsemigroup of some group reduces to that of so embedding $S$ in $Q(S)$ with the quotient topology. 
Theorem 2.2. Let $S$ be a cancellative topological semigroup such that $a S \cap b S \neq \varnothing$ for $a, b \in S$. Then $S$ is embeddable as a subsemigroup with nonempty interior in some topological group if and only if

(2.2) $Q(S)$ is a topological group with the quotient topology;

(2.3) $S$ is embeddable with nonempty interior in $Q(S)$ with this topology.

We first prove some lemmas.

Lemma 2.1. Let $G$ be a topological group. $G$ and $Q(G)$ are topologically isomorphic.

Proof. Since $\left[x y^{-1} p, p\right]=[x, y](x, y, p \in G)$ the equation (2.1) (with $S=G$ ) defines an isomorphism of $G$ onto $Q(G)$. Thus $G$ and $Q(G)$ are isomorphic and so, as pointed out above, $G$ and $Q(G)$ may be identified algebraically. It remains to show the equivalence of the two topologies: $\mathscr{T}_{1}$, the given topology of $G$, and $\mathscr{T}_{2}$, the quotient topology.

If $A \subset G, A \in \mathscr{T}_{2}$ iff $\left\{(x, y): x, y \in G\right.$ and $\left.x y^{-1} \in A\right\}$ is open in $G \times G$, iff $\left\{\left(x y^{-1}, y\right): x, y \in G\right.$ and $\left.x y^{-1} \in A\right\}$ is open in $G \times G\left((x, y) \rightarrow\left(x y^{-1}, y\right)\right.$ is a homeomorphism), i.e. $A \in \mathscr{T}_{2}$ iff $A \times G$ is open in $G \times G$ so that $A \in \mathscr{T}_{2}$ iff $A \in \mathscr{T}_{1}$.

Lemma 2.2. If $S$ is an open subsemigroup of a topological group $G$ and if $a S \cap b S \neq \varnothing$ for $a, b \in S$, then the topology which $Q(S)$ inherits as a subspace of $G$ is the quotient topology of $Q(S)$.

Proof. With $Q(S)=\left\{s t^{-1}: s, t \in S\right\}$ as above, let $\mathscr{T}$ be the topology which $Q(S)$ inherits from $G, \mathscr{Q}$ the quotient topology.

Let $A \subset Q(S), A \in \mathcal{Q}$. Then $A_{S}^{*}=\left\{(x, y): x, y \in S\right.$ and $\left.x y^{-1} \in A\right\}$ is open in $S \times S$ and so in $G \times G$ since $S$ is open in $G$. If $a b^{-1} \in A$, then

$$
a \in C=\left\{g: g \in G \text { and }(g, b) \in A_{s}^{*}\right\}=A b \cap S .
$$

Since $C$ is open in $G, C b^{-1}$ is open in $G$ and $a b^{-1} \in C b^{-1} \subset A$. Thus $A \in \mathscr{T}$, so that $\mathscr{Q} \subset \mathscr{T}$.

Let $A \subset Q(S), A \in \mathscr{T}$. Then $A=U \cap Q(S)$ where $U$ is open in $G$ and so, by Lemma 2.1, $U^{*}=\left\{(x, y): x, y \in G\right.$ and $\left.x y^{-1} \in U\right\}$ is open in $G \times G$. Thus $A_{S}^{*}=U^{*} \cap(S \times S)$ is open in $S \times S$, i.e. $A \in \mathscr{Q}$, so that $\mathscr{T} \subset \mathscr{Q}$.

Proof of Theorem 2.2. Suppose $S \subset G$ where $G$ is a topological group and $I=$ int $S \neq \varnothing$. (int $S$ denotes the interior of $S$.) Since $I$ is open in $G, S I$ is open in $G$ and, since $S I \subset S$, we have $S I \subset I$, so that $I$ is an ideal of $S$. Hence $I$ is an open subsemigroup of $G$. Let $a, b \in I$. By hypothesis there exist $s, t \in S$ such that $a s=b t$. Choose $j \in I$. Since $s j, t j \in I$, and $a(s j)=b(t j)$, $a I \cap b I \neq \varnothing$. Thus $I$ has a group of quotients $Q(I)$. In the case when $S$ is commutative it was shown by Peck (see for example (7), p. 197) that $Q(I)$ with the quotient topology is algebraically and topologically isomorphic to $Q(S)$ with the quotient topology and minor modifications show that the same E.M.S.-I 
is true in the present case. We therefore identify $Q(S)$ and $Q(I)$. Applying Lemma 2.2 to $I$, the quotient topology of $Q(S)$ is the topology which $Q(S)$ inherits as a subspace of $G$. Thus $Q(S)$ is a topological group with the quotient topology and $S$ is embeddable with non-empty interior in $Q(S)$ with this topology.

The reverse implication is obvious.

Corollary. Let $S$ be a cancellative topological semigroup such that $a S \cap b S \neq \varnothing$ for $a, b \in S$. If $Q(S)$ is a topological group with topology $\mathscr{T}$ and $S$ is embeddable with non-empty interior in $Q(S)$ with this topology, then $\mathscr{T}$ is the quotient topology.

Proof. This is immediate from the proof of Theorem 2.2 where it is pointed out that if $S$ is embeddable with non-empty interior in some topological group then the quotient topology of $Q(S)$ is the topology which $Q(S)$ inherits as a subspace of the group.

\section{Preliminary results}

This section consists of lemmas which isolate points which are required for the main embedding theorems and which may be of independent interest.

Lemma 3.1. Let $G$ be a group which is also a topological space. Then the following conditions are equivalent:

(1) $U$ an open subset of $G$ and $x \in G$ imply that $x U$ and $U x$ are open in $G$;

(2) the map $(x, y) \rightarrow x y$ of $G \times G$ into $G$ is separately continuous.

Proof. Suppose (1) holds. Fix $a \in G$. Let $b \in G$ and let $M$ be an open neighbourhood of $a b$. Then $N=a^{-1} M$ is an open neighbourhood of $b$ and $a b \in a N \subset M$, whence follows the continuity of $(x, y) \rightarrow x y$ in the second variable. Continuity in the first variable is similar.

Suppose (2) holds. Let $U \subset G$ be open and $x \in G$. Let $\left\{x_{\alpha}\right\}$ be a net in $G$ such that $x_{\alpha} \rightarrow x u$ for some $u \in U$. Using separate continuity, $x^{-1} x_{\alpha} \rightarrow u \in U$ which is open in $G$ and so $\left\{x^{-1} x_{\alpha}\right\}$ is eventually in $U$. It follows that $\left\{x_{\alpha}\right\}$ is eventually in $x U$ and $x U$ is open. Similarly $U x$ is open.

Lemma 3.2. Let $G$ be a group which is also a topological space and let $S \subset G$ be a non-empty open subsemigroup. If for fixed $g \in G$ the maps $x \rightarrow x g$ and $x \rightarrow g x$ of $G$ onto $G$ are continuous and if the map $(x, y) \rightarrow x y$ of $S \times S$ into $S$ is continuous, then the map $(x, y) \rightarrow x y$ of $G \times G$ onto $G$ is continuous.

Proof. Let $(a, b) \in G \times G$ and let $\left\{\left(a_{a}, b_{a}\right)\right\}$ be a net in $G \times G$ such that $\left(a_{\alpha}, b_{\alpha}\right) \rightarrow(a, b)$ in $G \times G$. Then $a_{\alpha} \rightarrow a$ and $b_{\alpha} \rightarrow b$ in $G$. Using separate continuity of multiplication on $G, a^{-1} a_{\alpha} \rightarrow e$ and $b_{a} b^{-1} \rightarrow e$ where $e$ is the identity of $G$ and, if $p, q \in S, p a^{-1} a_{\alpha} \rightarrow p$ and $b_{\alpha} b^{-1} q \rightarrow q$. Since $S$ is open in $G,\left\{p a^{-1} a_{\alpha}\right\}$ and $\left\{b_{\alpha} b^{-1} q\right\}$ are eventually in $S$. Joint continuity of multiplication in $S$ gives $p a^{-1} a_{\alpha} b_{\alpha} b^{-1} q \rightarrow p q$ in $S$ and so in $G$ whence, by repeated use of the separate continuity of multiplication in $G, a_{\alpha} b_{\alpha} \rightarrow a b$. Thus the result is established. 
Lemma 3.3. Let $G$ be a group with a Hausdorff topology and separately continuous multiplication. Suppose $G$ contains a non-empty open subset $S$ such that $S$ is locally compact in the relative topology. Then $G$ is a locally compact topological group.

Proof. Let $a \in G$ and let $N$ be an open neighbourhood of $a$. If $s \in S$, $s a^{-1} N$ is an open neighbourhood of $s$ by Lemma 3.1 and so $M=s a^{-1} N \cap S$ is an open neighbourhood of $s$ both in $S$ and in $G$. Since $S$ is locally compact in the relative topology, there exists a compact neighbourhood $C$ of $s$ in $S$ such that $s \in C \subset M$. Now $S$ is open in $G$ and so $C$ is a compact neighbourhood of $s$ in $G$. It follows from Lemma 3.1 that $a \in a s^{-1} C \subset N$ and $a s^{-1} C$ is a compact neighbourhood of $a$. The topology of $G$ is thus locally compact. Since $G$ is a group with a locally compact Hausdorff topology and separately continuous multiplication, it follows from a result of Ellis in (1) (see Theorem 4.3 below) that $G$ is a locally compact topological group.

\section{The embedding theorems}

Let $S$ be a topological semigroup. For $s \in S, L_{s}$ and $R_{s}$ are the maps of $S$ into $S$ defined by $L_{s}(x)=s x$ and $R_{s}(x)=x s(x \in S)$. For $A \subset S$ and $s \in S$, $A_{-1} s=\{t: t \in S$ and $a t=s$ for some $a \in A\}$. It should be noted that if $S$ is a topological group, $A_{-1} s=A^{-1} s$ where $A^{-1}=\left\{a^{-1}: a \in A\right\}$.

We now state and prove our main results.

Theorem 4.1. Let $S$ be a topological semigroup such that $a S \cap b S \neq \varnothing$ for $a, b \in S$. Then $S$ is embeddable as an open subsemigroup of a topological group if and only if

(4.1) $S$ is cancellative;

(4.2) for all $x, y \in S$ and all open subsets $U$ of $S, x U, U x$ and $(U y)_{-1} x$ are open in $S$.

We first prove a technical lemma.

Lemma 4.1. Let $S$ be a cancellative semigroup such that $a S \cap b S \neq \varnothing$ for $a, b \in S$. If $p, q, r, s \in S$ and $V \subset S$, then there are functions $v \rightarrow v^{\prime}, v \rightarrow \bar{v}$ defined on $V$ to $S$ such that

$$
\begin{aligned}
& \left(p q^{-1} V r s^{-1}\right) \cap S=\bigcup_{v \in V}\left(R_{s v^{\prime}}\right)^{-1}\left(L_{p}\left[L_{q}^{-1}\left(V r v^{\prime}\right)\right]\right) \\
& \left(p q^{-1} V^{-1} r s^{-1}\right) \cap S=\bigcup_{v \in V}\left(R_{s \bar{v}}\right)^{-1}\left(L_{p}\left[(V q)_{-1}(r \bar{v})\right]\right) .
\end{aligned}
$$

Proof. Choose $v \in V$. Since $q, r \in S$ are fixed, and since $q S \cap v r S \neq \varnothing$, there exist $v^{\prime}, v^{\prime \prime} \in S$ such that $v r v^{\prime}=q v^{\prime \prime}$. Choose and fix such a $v^{\prime}, v^{\prime \prime}$ for each $v \in S$. Similarly fix $\bar{v}, \bar{v} \in S$ such that $v q \bar{v}=r \bar{v}$.

If $x \in\left(p q^{-1} V r s^{-1}\right) \cap S$, then $x \in S$ and $x=p q^{-1} v r s^{-1}$ for some $v \in V$ so that $(x s)(v r)^{-1}=p q^{-1}$. Since $v r v^{\prime}=q v^{\prime \prime}$, it follows from the equivalence 
relation which defines $Q(S)$ that $(x s) v^{\prime}=p v^{\prime \prime}$. The equation $v r v^{\prime}=q v^{\prime \prime}$ also shows that $v^{\prime \prime} \in L_{q}^{-1}\left(V r v^{\prime}\right)$ and so $x \in\left(R_{s v^{\prime}}\right)^{-1}\left(L_{p}\left[L_{q}^{-1}\left(V r v^{\prime}\right)\right]\right)$. If

$$
x \in \bigcup_{v \in V}\left(R_{s v^{\prime}}\right)^{-1}\left(L_{p}\left[L_{q}^{-1}\left(V r v^{\prime}\right)\right]\right)
$$

then $x s v^{\prime}=p y$ where $q y=w r v^{\prime}$ for some $w \in V$. By the equivalence relation which defines $Q(S),(x s)(w r)^{-1}=p q^{-1}$, so that $x \in\left(p q^{-1} V r s^{-1}\right) \cap S$. Thus (4.3) is established.

If $x \in\left(p q^{-1} V^{-1} r s^{-1}\right) \cap S$, then $x \in S$ and $x=p q^{-1} v^{-1} r s^{-1}$ for some $v \in V$. Thus $(x s) r^{-1}=p(v q)^{-1}$ and, since $v q \bar{v}=r \bar{v}$, we have $p \bar{v}=x s \bar{v}$. Since $\bar{v} \in(V q)_{-1}(r \bar{v})$, we have $x \in\left(R_{s \bar{v}}\right)^{-1}\left(L_{p}\left[(V q)_{-1}(r \bar{v})\right]\right)$. If

$$
x \in \bigcup_{v \in V}\left(R_{s \bar{v}}\right)^{-1}\left(L_{p}\left[(V q)_{-1}(r \bar{v})\right]\right) \text {, }
$$

then $x s \bar{v}=p y$ where $w q y=r \bar{v}$ for some $w \in V$. Hence $(x s) r^{-1}=p(w q)^{-1}$, i.e. $x=p q^{-1} w^{-1} r s^{-1} \in\left(p q^{-1} V^{-1} r s^{-1}\right) \cap S$. This proves (4.4).

Proof of Theorem 4.1. Suppose conditions (4.1) and (4.2) hold and let $Q(S)$ be the group of quotients of $S$ in which the image of $S$ is identified with $S$ as already described. We first of all define a topology for $Q(S)$. It follows from the corollary to Theorem 2.2 that this topology is the quotient topology of $Q(S)$ but we justify its use by pointing out that it is more easily manipulated. Define

(4.5) $\mathscr{T}=\left\{A: A \subset Q(S)\right.$ and $\left(p q^{-1} A r s^{-1}\right) \cap S$ is open in $S$ for all $\left.p, q, r, s \in S\right\}$.

It is clear that $Q(S), \varnothing \in \mathscr{T}$ and for $A, B \in \mathscr{T}, p, q, r, s \in S$,

$\left[p q^{-1}(A \cap B) r s^{-1}\right] \cap S=\left[\left(p q^{-1} A r s^{-1}\right) \cap S\right] \cap\left[\left(p q^{-1} B r s^{-1}\right) \cap S\right]$ so that $A \cap B \in \mathscr{T}$.

It follows similarly that the union of an arbitrary family of members of $\mathscr{T}$ is again in $\mathscr{T}$. Thus $\mathscr{T}$ is a topology for $Q(S)$ and for the rest of the proof $Q(S)$ will denote the group of quotients with topology $\mathscr{T}$.

The first part of our proof consists of showing that $S$ is an open subsemigroup of $Q(S)$.

(4.6) Left and right translations in $Q(S)$ are open maps. If $A \in \mathscr{T}$ and $u v^{-1} \in Q(S)$, then for any $p, q, r, s \in S, p q^{-1} u v^{-1}=w z^{-1} \in Q(S)$ so that

$$
\left[p q^{-1}\left(u v^{-1} A\right) r s^{-1}\right] \cap S=\left(w z^{-1} A r s^{-1}\right) \cap S
$$

which is open in $S$. Hence $u v^{-1} A \in \mathscr{T}$ and similarly $A u v^{-1} \in \mathscr{T}$.

(4.7) Multiplication in $Q(S)$ is separately continuous. This is immediate from (4.6) and Lemma 3.1.

Let $\mathscr{T}^{*}$ be the topology which $S$ inherits from $Q(S)$ and $\mathscr{S}$ be the original topology of $S$.

(4.8) If $A \subset Q(S)$ and $A \in \mathscr{T}$ then $A \cap S \in \mathscr{S}$. If $A \in \mathscr{T}$, then in particular $\left(p p^{-1} A p p^{-1}\right) \cap S \in \mathscr{S}$ for any $p \in S$ and so $A \cap S \in \mathscr{S}$. 
(4.9) If $A \subset S$ and $A \in \mathscr{S}$, then $A \in \mathscr{T}$. If $p, q, r, s \in S$, it follows from (4.3) that $\left(p q^{-1} A r s^{-1}\right) \cap S=\bigcup_{a \in A}\left(R_{s a^{\prime}}\right)^{-1}\left(L_{p}\left[L_{q}^{-1}\left(A r a^{\prime}\right)\right]\right) \in \mathscr{S}$ by (4.2) and the continuity of multiplication in $S$, i.e. (4.9) holds.

It is immediate from (4.8) and (4.9) that $\mathscr{T}^{*}=\mathscr{S}$ and that $S$ is an open subsemigroup of $Q(S)$. We now show that $Q(S)$ is a topological group.

(4.10) $\mathscr{T}$ is a Hausdorff topology. Let $s t^{-1}, w z^{-1} \in Q(S), s t^{-1} \neq w z^{-1}$. Choose $x, y \in S$ such that $t x=z y$. By the defining equivalence relation for $Q(S), s x \neq w y$. Since $S$ is Hausdorff, there exist disjoint open neighbourhoods $U$ and $V$ in $S$ of $s x$ and $w y$ respectively. It follows from (4.9) that $U, V \in \mathscr{T}$ and then an application of (4.6) shows that $U x^{-1} t^{-1}$ and $V x^{-1} t^{-1}$ are disjoint neighbourhoods of $s t^{-1}$ and $w z^{-1}$ in $Q(S)$.

(4.11) Multiplication in $Q(S)$ is jointly continuous. Since $S$ is an open subsemigroup of $Q(S)$, this result follows from (4.7) and Lemma 3.2.

(4.12) The inverse map is continuous in $Q(S)$. Let $A$ be a subset of $S$ with $A \in \mathscr{T}$. If $p, q, r, s \in S,\left(p q^{-1} A^{-1} r s^{-1}\right) \cap S=\bigcup_{a \in A}\left(R_{s \bar{a}}\right)^{-1}\left(L_{p}\left[(A q)_{-1}(r \bar{a})\right]\right)$ by (4.4) and so $A^{-1} \in \mathscr{T}$, using (4.2) and continuous multiplication in $S$. Let $N$ be a subset of $Q(S)$ such that $N \in \mathscr{T}$ and let $x y^{-1} \in N$. The set $A=N y \cap S \subset S$ and, by (4.6), $A \in \mathscr{T}$. As above $A^{-1} \in \mathscr{T}$. Now $x^{-1} \in A^{-1}$ so that $\left(x y^{-1}\right)^{-1}=y x^{-1} \in y A^{-1} \subset N^{-1}$. Since $y A^{-1} \in \mathscr{T}, N^{-1} \in \mathscr{T}$.

Equations (4.10)-(4.12) combine to show that $Q(S)$ is a topological group, establishing one half of the theorem.

Now suppose that $S \subset G$ where $G$ is a topological group and $S$ is an open subsemigroup of $G$. It is immediate that (4.1) is satisfied.

Let $U \subset S$ be open in $S$ and let $x, y \in S$. Since $S$ is open in $G, U$ is open in $G$, so that $U x$ and $x U$ are open in $G$. But $U x, x U \subset S$ and so $U x$ and $x U$ are open in $S$. Now $U y$ open in $G$ implies that $(U y)^{-1}$ is open in $G$ and

$$
(U y)_{-1} x=(U y)^{-1} x \cap S .
$$

Since $(U y)^{-1} x$ is open in $G,(U y)_{-1} x$ is open in $S$, showing that (4.2) holds.

Note. If in the above theorem we make the additional assumption that $S$ is commutative the labour of the proof is to some extent reduced. In particular, $\mathscr{T}$ consists of those sets $A \subset Q(S)$ such that $\left(p q^{-1} A\right) \cap S$ is open in $S$ for all $p, q \in S$ and equations (4.3) and (4.4) reduce to

$$
\begin{aligned}
& \left.\left(p q^{-1} V\right) \cap S=\left(L_{q}\right)^{-1}\left(L_{p}\right)(V)\right) \text { and } \\
& \left(p q^{-1} A^{-1}\right) \cap S=(A q)_{-1} p .
\end{aligned}
$$

We now prove a theorem on embedding topological semigroups with non-empty interior in topological groups. It is independent of the above theorem but is phrased so that they may be combined to give explicit conditions for embedding with non-empty interior. 
Theorem 4.2. Let $S$ be a topological semigroup such that $a S \cap b S \neq \varnothing$ for $a, b \in S$. Then $S$ is embeddable with non-empty interior in a topological group if and only if

(4.1) $S$ is cancellative;

(4.13) there exists an open ideal $I$ in $S$ and $i_{0} \in I$ such that

(4.13.1) I is embeddable as an open subsemigroup of a topological group,

(4.13.2) for any $x \in S$ and any open neighbourhood $U$ of $x$ there exists an open neighbourhood $A$ of $i_{0}$ with $A \subset I$ and $\left(L_{i_{0}}\right)^{-1}(A x) \subset U$.

Proof. Suppose (4.1) and (4.13) hold with $I$ embeddable as an open subsemigroup of a topological group $G$. Then, as in the proof of Theorem 2.2, $I$ has a group of quotients $Q(I)$ which is isomorphic to $Q(S)$ and $Q(I) \subset G$. We may therefore identify $S$ algebraically with a subset of $G$ and it remains to show that this embedding is also topological.

We first of all show that for any $x \in S$ and any open set $A \subset I, A x$ is open in $S$. Choose $x \in S$ and any set $A \subset I$ which is open in $S$. Since $I$ is an open subsemigroup of $G, A$ is open in $G$ and so, since $x \in G, A x$ is open in $G$. Hence $A x \subset I$. From the embedding of $I$ in $G$ and the openness of $I$ in $G, A x$ is open in $I$ and so in $S$.

Suppose $\left\{x_{\alpha}\right\}$ is a net in $S$ such that $x_{\alpha} \rightarrow x \in S$ in the topology of $S$. Since $i_{0} \in I$ which is an ideal of $S,\left\{i_{0} x_{\alpha}\right\}$ is a net in $I$ with $i_{0} x_{\alpha} \rightarrow i_{0} x \in I$ in the topology which $I$ inherits from $S$. It follows from (4.13) that $i_{0} x_{\alpha} \rightarrow i_{0} x$ in the topology of $G$ which is a topological group and so $x_{\alpha} \rightarrow x$ in the topology of $G$.

Let $\left\{x_{\alpha}\right\}$ be a net in $S$ such that $x_{\alpha} \rightarrow x \in S$ in the topology which $S$ inherits from $G$. Then $x_{\alpha} \rightarrow x$ in $G$ and so $i_{0} x_{\alpha} \rightarrow i_{0} x \in I$ in the topology which $I$ inherits from $G$. By (4.13) this means that $i_{0} x_{\alpha} \rightarrow i_{0} x$ in the topology of $S$. Let $U$ be an open neighbourhood of $x$ in $S$. An application of (4.13.2) produces an open neighbourhood $A$ of $i_{0}$ in $S$ with $A \subset I$ and $\left(L_{i_{0}}\right)^{-1}(A x) \subset U$. From above $A x$ is an open neighbourhood of $i_{0} x$ in $S$. Hence $\left\{i_{0} x_{\alpha}\right\}$ is eventually in $A x$ and $\left\{x_{\alpha}\right\}$ is eventually in $\left(L_{i_{0}}\right)^{-1}(A x) \subset U$. Thus $x_{\alpha} \rightarrow x$ in the topology of $S$.

The above calculations show that the topology of $S$ is in fact the topology which $S$ inherits from $G$. Since $I \subset S$ and $I$ is open in $G, S$ is embeddable with non-empty interior in the topological group $G$.

We now consider $S$ contained in a topological group $G$ with $I=$ int $S$ non-empty in $G$. It is immediate that (4.1) is satisfied. As in the proof of Theorem 2.2, $I$ is an open ideal of $S$ and $I$ is an open subsemigroup of $G$. Thus (4.13.1) holds. Choose $i_{0} \in I$. We show that $i_{0}$ satisfies (4.13.2). For $x \in S$ and $U$ an open neighbourhood of $x$ in $S, U=V \cap S$ where $V$ is an open subset of $G$. Now $A=\left(i_{0} V x^{-1}\right) \cap I$ is an open neighbourhood of $i_{0}$ in $S$ and $\left(L_{i_{0}}\right)^{-1}(A x) \subset U$, establishing (4.13.2). 
We now consider locally compact semigroups. The assumption of local compactness simplifies Theorem 4.1, principally due to the following result of Ellis (1).

Theorem 4.3. Let $G$ be a group with a locally compact Hausdorff topology. If multiplication is separately continuous, then $G$ is a topological group.

The following theorem generalises a result in (2) where complete separable metric topological semigroups are considered.

Theorem 4.4. Let $S$ be a locally compact topological semigroup such that $a S \cap b S \neq \varnothing$ for $a, b \in S$. Then $S$ is embeddable as an open subsemigroup of $a$ topological group if and only if

(4.1) $S$ is cancellative;

(4.14) for all $x \in S$ and all open subsets $U$ of $S, x U$ and $U x$ are open in $S$. When $S$ is thus embeddable, the group is locally compact.

Proof. Since the proof is basically the same as that of Theorem 4.1, we refer to this proof and indicate the differences.

Suppose (4.1) and (4.14) hold. Then $Q(S)$ has the topology $\mathscr{T}$ defined by (4.5) and (4.6)-(4.9) stand. It follows that $S$ is an open subsemigroup of $Q(S)$. Since (4.10) also holds and $S$ is locally compact in the relative topology, an application of Lemma 3.3 shows that $Q(S)$ is a locally compact topological group. It is in the proof of Lemma 3.3 that Theorem 4.3 is used.

Suppose $S$ is embeddable as an open subsemigroup of a topological group $G$. Theorem 4.1 shows that (4.1) and (4.14) are satisfied and it follows from Lemma 3.3 that $G$ is locally compact.

\section{Examples}

The purpose of this section is to illustrate some of the preceding results and delineate the lack of possibilities for removal of some of our embedding conditions. We first quote a theorem of Schieferdecker (9) which we use to justify two of the examples.

Theorem 5.1. Let $S$ be a cancellative topological semigroup such that $a S \cap b S \neq \varnothing$ for $a, b \in S$. Then

(5.1) $Q(S)$ may be topologised to be a topological group and

(5.2) $S$ is embeddable in this topological group if and only if

(5.3) the topology of $S$ is derived from a uniformity;

(5.4) this uniformity is defined by a basis $\mathscr{B}$ whose elements $B$ satisfy the conditions

(5.4.1) $(a, b) \in B$ implies that $(a x, b x) \in B$ for $x \in S$;

(5.4.2) $(a x, b x) \in B$ implies that $(a, b) \in B$ for $x \in S$; 
(5.4.3) for $y \in S$ and $B \in \mathscr{B}$ there exists $B^{\prime}=B^{\prime}(y, B) \in \mathscr{B}$ so that $(a, b) \in B^{\prime}$ implies that $(y a, y b) \in B$;

(5.4.4) for $y \in S$ and $B \in \mathscr{B}$ there exists $B^{\prime \prime}=B^{\prime \prime}(y, B) \in \mathscr{B}$ so that $(y a, y b) \in B^{\prime \prime}$ implies that $(a, b) \in B$.

Corollary. Let $S$ be a cancellative topological semigroup such that $a S \cap b S \neq \varnothing$ for $a, b \in S$. Then $S$ is embeddable in a topological group if and only if $S$ satisfies (5.3) and (5.4).

Proof. If $S$ satisfies (5.3) and (5.4), then it follows that (5.1) and (5.2) hold, establishing the implication in one direction.

If $S$ is embeddable in a topological group $G$, then $Q(S) \subset G$ and $Q(S)$ is a topological group with the topology inherited from $G$ so that (5.1) and (5.2) hold. Hence, by the theorem, (5.3) and (5.4) hold.

It is this corollary which we shall use in the examples which follow.

Example 5.1. This example is chosen to exhibit a simple topological semigroup which is not embeddable in a topological group.

Let $S=[0, \infty)$ with addition as operation. Let $[0,1]$ have the discrete topology and $(1, \infty)$ the usual topology from $\mathbf{R}$ (the real numbers). Then $S$ is a commutative cancellative topological semigroup. Since $S$ is locally compact Hausdorff, the topology of $S$ is generated by a uniformity with a base $\mathscr{B}$. The discreteness of the topology of $S$ in $[0,1]$ means that there exists $B^{\prime} \in \mathscr{B}$ such that $B^{\prime} \cap([0,1] \times[0,1])=\Delta \cap([0,1] \times[0,1])$ where $\Delta=\{(s, s): s \in S\}$. If $S$ is embeddable in some topological group, then $\mathscr{B}$ must satisfy (5.4.2), and so it follows that $B^{\prime}=\Delta$, which implies that $S$ is discrete. Thus $S$ is not embeddable in any topological group by the corollary to Theorem 5.1.

That $S$ is not embeddable in $Q(S)$ with the quotient topology is pointed out by $\mathrm{J}$. H. Williamson in (11). It is of interest that Williamson uses this semigroup as an example of a topological semigroup with a nonhomogeneous topology. Since $S$ is locally compact it is precisely this nonhomogeneity which prevents its being embeddable as is shown in Theorem 4.4.

Example 5.2. The hypothesis in the corollary to Theorem 2.2 that $S$ have non-empty interior cannot be dropped. This is illustrated by the following example.

Let $G$ be the positive rationals with the topology inherited from the usual topology of $\mathbf{R}$ and multiplication as operation. Let $S$ be the positive integers. Then $S$ is embeddable with empty interior in $G$. We have, however, that $S$ is embeddable in $Q(S)$ with the quotient topology, i.e. in the positive rationals with the discrete topology. Hence $S$ is embeddable in its group of quotients with two different topologies.

Example 5.3. We now construct a cancellative commutative topological semigroup in which translation is an open map but which is not embeddable 
in any topological group. This shows that the conditions of the embedding theorems cannot be reduced. This semigroup also provides a counterexample to Theorem 3.2.14 of (5).

Let $S=[0, \infty)$ with addition as the operation. Then $S$ is a commutative cancellative semigroup with $Q(S)=\mathbf{R}$ and $\mathscr{B}=\{[a, b): a, b \in S\}$ is a base for a topology $\mathscr{T}$ for $S$. With this topology $S$ is easily shown to be a topological semigroup. Since the members of $\mathscr{B}$ are both open and closed, the characteristic function of a member of $\mathscr{B}$ is continuous and so $\mathscr{T}$ is completely regular. Hence $\mathscr{T}$ is generated by a uniformity $\mathscr{U}$ having base $\mathscr{C}$. If $S$ is embeddable in some topological group, the corollary to Theorem 5.1 shows that (5.4.1) holds for $\mathscr{C}$. Choose any $a \in S, a>0$. If $b>a$, then $[a, b)$ is an open neighbourhood of $a$. Thus there exists $C \in \mathscr{C}$ such that $C[a] \subset[a, b)$. It follows from (5.4.1) that $C \cap\{(x, y): x, y \in S$ and $x<y<a\}=\varnothing$. Since $C \in \mathscr{C}$ there exists $D \in \mathscr{C}$ with $D \subset C^{-1}$, and $D \cap\{(x, y): x, y \in S$ and $a>x>y\}=\varnothing$. Hence, for $x \in S \cap[0, a)$, if $(x, y) \in C \cap D \in \mathscr{U}$, then $x=y$ and so $\{x\}$ is open. Since $a \in S$ was arbitrary, this shows that $S$ is discrete, which is a contradiction. Thus $S$ is not embeddable in a topological group.

We now note that translation in $S$ is an open map and that $S$ is cancellative and commutative. Theorem 4.1 does not apply since $[1,2)_{-1} 3=(1,2] \notin \mathscr{T}$. If $S$ were locally compact, then some $[a, b)$ would be compact. It is, however, clear that the family $\left\{\left[a, b-\frac{1}{n}\right): n\right.$ a positive integer $\}$ covers $[a, b)$ but has no finite subcover. Hence $S$ is not locally compact and Theorem 4.4 is therefore inapplicable.

\section{REFERENCES}

(1) R. ElLIs, Locally compact transformation groups, Duke Math. J. 24 (1957), 119-125.

(2) B. Gelbaum, G. K. Kalisch and J. M. H. Olmsted, On the embedding of topological semigroups and integral domains, Proc. Amer. Math. Soc. 2 (1951), 807-821.

(3) A. Malcev, On the immersion of an algebraic ring into a field, Math. Ann. 113 (1937), 686-691.

(4) O. ORe, Linear equations in noncommutative fields, Ann. of Math. 32 (1931), 463-477.

(5) A. B. PaAlman-de-Mranda, Topological Semigroups (Math. Centrum, Amsterdam, 1964).

(6) J. E. L. PECK, The embedding of a topological semigroup in a topological group and its generalisations (Ph.D. Dissertation, Yale University, 1950).

(7) N. J. Rothman, Embedding of topological semigroups, Math. Ann. 139 (1960), 197-203. 
(8) E. SCHIEFERDECKER, Zur Einbettung metrischer Halbgruppen in ihre Quotientenhalbgruppen, Math. Z. 62 (1955), 443-468.

(9) E. SCHIEFERDECKer, Einbettungssätze für topologische Halbgruppen, Math. Ann. 131 (1956), 372-384.

(10) D. TAMARI, Sur l'immersion d'un semi-groupe topologique dans un groupe topologique, Colloques Internationaux du Centre National de la Recherche Scientifique, Algèbre et Théorie des Nombres 24 (1950), 217-221.

(11) J. H. Williamson, Harmonic analysis on semigroups, J. London Math. Soc. 42 (1967), 1-41.

UNIVERSITY OF ABERDEEN 